\title{
One-stage thumb lengthening with use of an osteocutaneous 2nd metacarpal flap
}

\author{
Panagiotis Givissis • Stavros I. Stavridis • \\ Konstantinos Ditsios • Anastasios Christodoulou
}

Received: 3 August 2009/ Accepted: 11 November 2009/Published online: 26 November 2009

(C) Springer-Verlag 2009

\begin{abstract}
Traumatic thumb amputation represents an extremely disabling entity, thus rendering its reconstruction a procedure of paramount importance. A case of a patient, who sustained a traumatic amputation of his left index finger at the metacarpophalangeal joint and of his left thumb in the middle of the proximal phalanx 4 months ago and was initially treated elsewhere, is described. For the thumb reconstruction, an osteocutaneous flap of the radial side of the 2nd metacarpal, which consisted of a 3, 5-cm bony segment with the overlying skin and its blood and nerve supply was used. The flap was transferred and fixed with a plate and screws to the palmar-medial side of the stump of the thumb, while the 1 st web space was deepened by removing the rest of the second metacarpal, while a partial skin graft was used to cover a remaining gap. Thumb functionality was restored immediately postoperatively, and the overall result was satisfactory.
\end{abstract}

Keywords Thumb amputation - Thumb reconstruction . Osteocutaneous flap $\cdot$ Metacarpal $\cdot$ Web space deepening

P. Givissis - S. I. Stavridis - K. Ditsios - A. Christodoulou 1st Orthopaedic Department of Aristotle University of Thessaloniki, "G. Papanikolaou" General Hospital, 57010 Exohi, Thessaloniki, Greece

P. Givissis $(\bowtie)$

9 Papanikolaou Str., Panorama, Mail Box 215,

55210 Thessaloniki, Greece

e-mail: givissis@otenet.gr
On the length, strength, free lateral motion and perfect mobility of the thumb depends the power of the human hand. Sir Charles Bell, 1833 [1].

\section{Introduction}

The human thumb represents undoubtedly the most important digit, since its opposition ability greatly separates humans from lower primates, while it accounts for almost half or even more of hand functionality [2, 3]. Hence, traumatic amputation of the thumb constitutes an extremely severe disability, thus rendering its adequate management an act of paramount importance.

Position, stability, strength, length, motion, sensibility and appearance are the attributes that make the thumb unique. Of these qualities, the first four must be present to an acceptable extent for function to approach normality, while the latter three are very desirable but not essential [4].

In the majority of the cases, thumb reconstruction is performed as a secondary procedure [5]. The indication and the selection of the method depend upon the level of amputation, the dominance of the injured hand, and the presence of other injuries to the same hand as well as age, sex, occupation, and intelligence of the patient [5]. According to different indications, a great variety of procedures have been described, such as autologous transplantation of the great or second toe (toe to thumb) [6-8], use of several types of flaps, simple or vascular, originating from the digits up to the forearm, widening of the first web space with Z-plasties and elongation of the 1st metacarpal by distraction osteogenesis [9].

We report a case of an osteocutaneous flap from the second metacarpal that was used for amputated thumb reconstruction, a modification of Loda's Vascular Rein Technique [10]. 


\section{Case report}

A 56-year-old manual worker had sustained a traumatic amputation of the left index finger at the level of the metacarpophalangeal joint, and of the left thumb in the middle of the proximal phalanx, with additional injury of the distal phalanx of the left ring finger, during a work accident with a ribbon saw 4 months ago. Immediately post injury, the patient was admitted to a regional hospital, where the hand was cleaned up and stump closure was performed. Upon his reference to us, surgical wound healing was satisfactory, and there were no signs of further lesions such as infection or neuroma formation in his hand (Fig. 1a, b).

Based on the concept of restoring thumb sensibility [11] and the fact that the useless second metacarpal stump should be removed in order to deepen the 1st web space, we modified Loda's Vascular Rein Technique [10], utilizing an osteocutaneous 2nd metacarpal flap for thumb reconstruction.
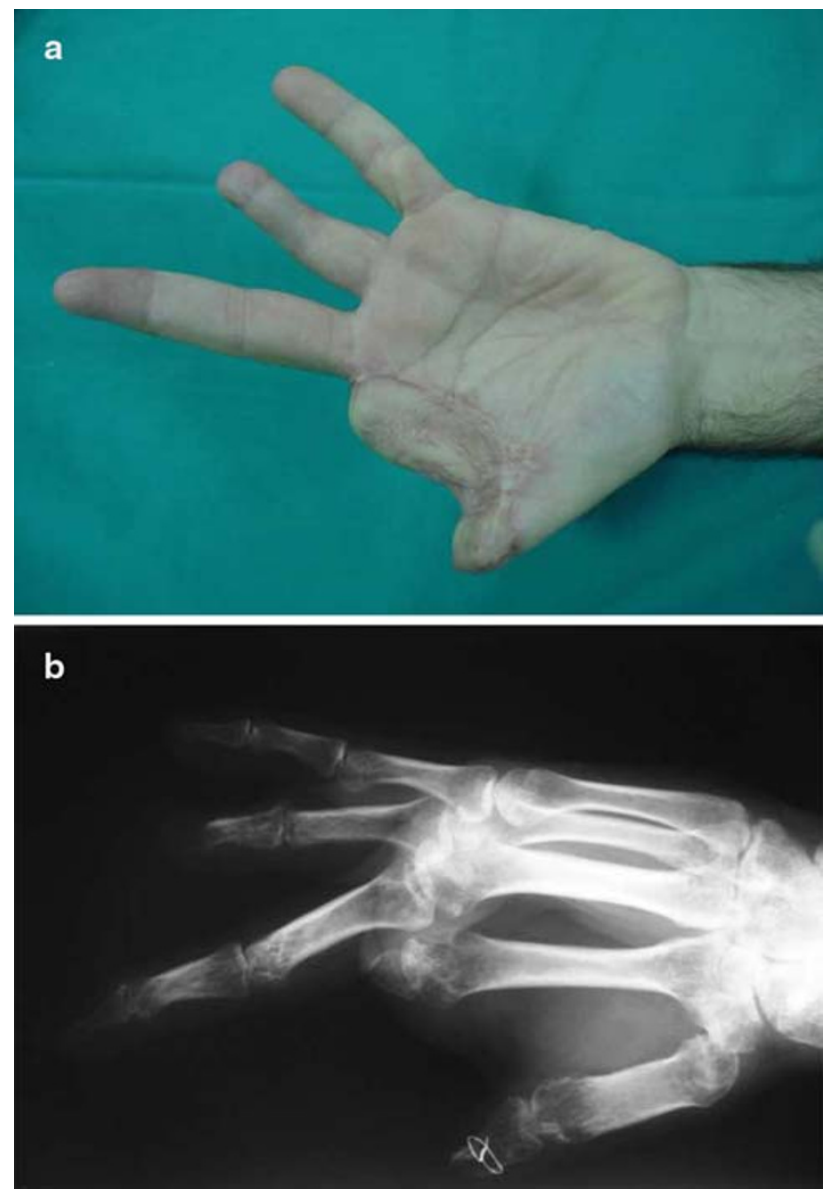

Fig. 1 Preoperative clinical (a) and radiological picture of the amputated hand
Detailed preoperative planning and drawing of the flap (Fig. 2a), were followed by a dorsal approach and thorough tissue preparation. A longitudinal osteotomy of the 2nd metacarpal starting at its distal end was performed with a saw blade. An osseous fragment of the radial side of the 2nd metacarpal with a length of ca $3.5 \mathrm{~cm}$ was prepared in that way.

Apart from the metacarpal bony fragment, the osteocutaneous flap also contained the overlying skin which is perfused by a branch of the radial artery and innervated by a branch of the superficial radial nerve (Fig. 2b, c).

The remaining second metacarpal was removed at a level just distal to the 2nd carpometacarpal joint. The flap was transferred to the palmar-medial side of the thumb stump that had been adequately prepared before, through an extension of the skin incision (Fig. 2d). An osteosynthesis by using a special plate and screws (Profyle Small Bone Plating System, Stryker ${ }^{\circledR}$, MI, USA) was performed (Fig. 2e).

Having already removed the remaining 2nd metacarpal, first web space deepening was completed by extending the palmar skin incision and developing a flap that was transferred on the dorsal skin defect of the first web space (Fig. 3a). In order to cover a small remaining gap in the palmar side of the first web space, a free partial skin graft that had been harvested from the palmar surface of the forearm was applied (Fig. 3b).

Antibiotics were administered for 3 days following surgery, and the sutures were removed after 2 weeks. The patient's postoperative course was generally uneventful both in terms of wound healing and survival of the flap and the graft.

Immediately postoperatively, a restoration of the hand's grip function was achieved. The thumb demonstrated a satisfactory mobility due to retention of the muscle's insertions at the base of the 1st phalanx, while sensation was also preserved (Fig. 3c). At the last follow-up examination 14 months postoperatively, the patient scored 16 points in the DASH score and 8 points in the Kapandji score (Fig. 4a, b). He reported complaints due to the screws throughout gripping of objects.

\section{Discussion}

Traumatic thumb amputation constitutes a devastating injury with dramatic consequences in patient's everyday life, since it is generally accepted that the thumb represents more than $40 \%$ of hand function [3].

A variety of surgical techniques for thumb reconstruction have been described in the literature, according to various indications, among which the level of amputation is the most important. In cases similar to our case, where the 

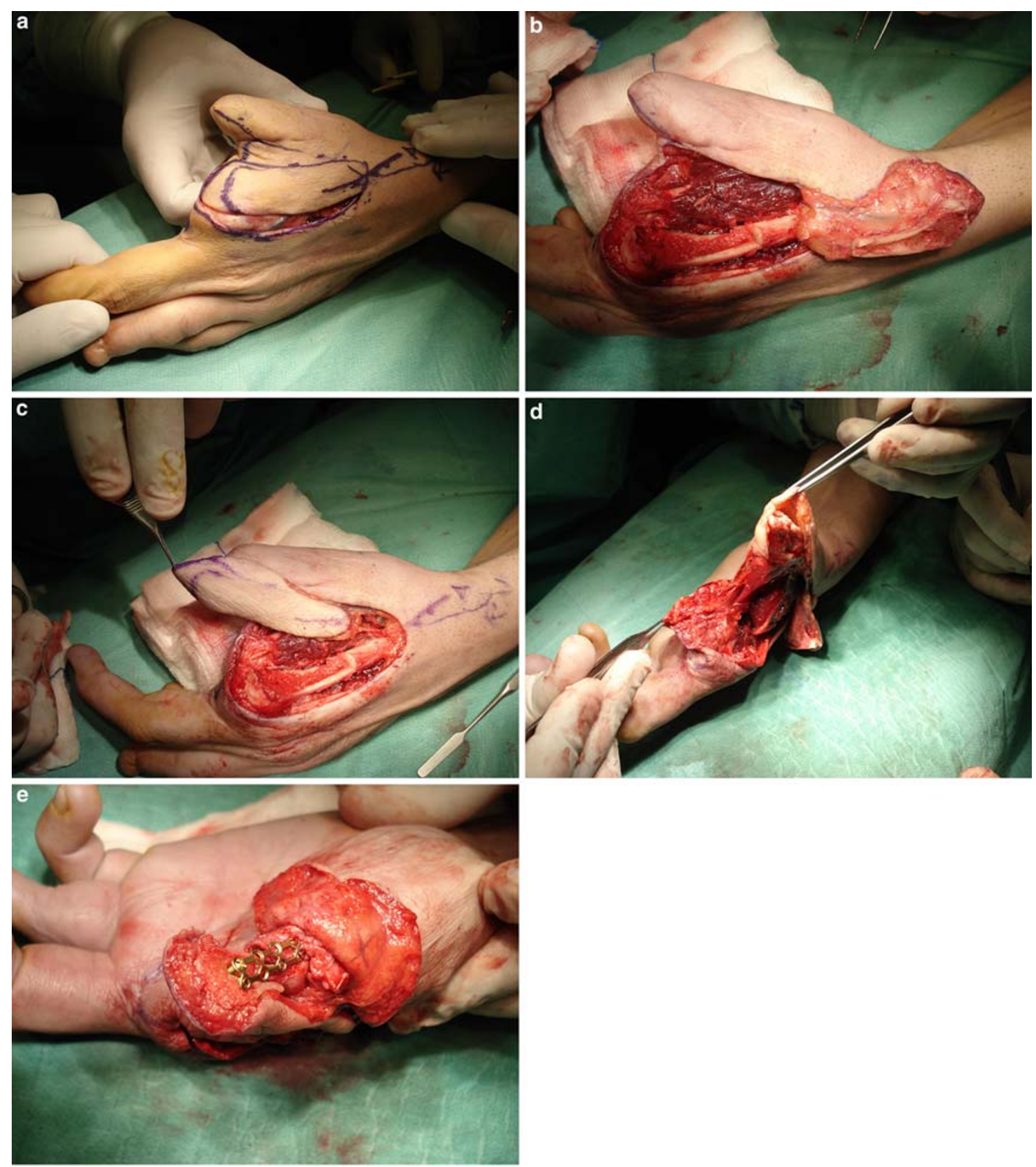

Fig. 2 After detailed flap-drawing (a) the 2nd metacarpal osteocutaneous flap (b, c) and thumb stump (d) were prepared followed by transfer and osteosynthesis (e)

amputation level is at the proximal half of the middle third, an elongation of the ray of the thumb is necessary. Alternative options are the application of distraction osteogenesis, autologous toe transplantation by use of microsurgical techniques and the use of parts originating from other injured digits for pollicisation (spare part technique) [12].

While metacarpal lengthening via distraction osteogenesis can achieve a mean elongation of $3.5 \mathrm{~cm}$, it bears the significant drawback that the full treatment lasts several months with continuous application of the external fixator [9].

Another valuable option for amputated thumb reconstruction is the autologous transplantation of the great or the second toe, which is a demanding and time consuming procedure. Moreover, this method bears the disadvantage of significant donor site morbidity, especially when the great toe has been used, while several weeks are required until neothumb sensation and mobility are restored $[2,4]$. 

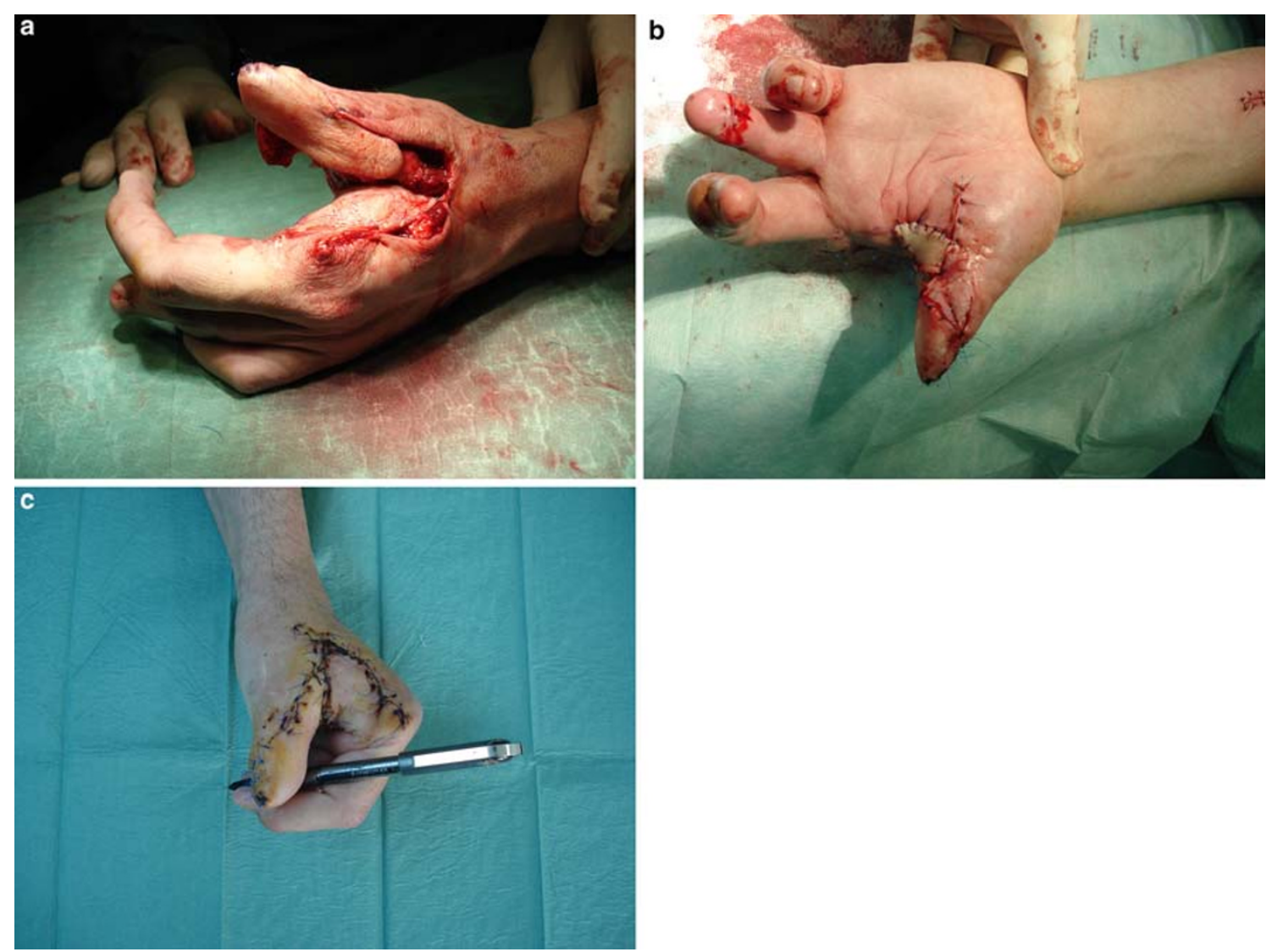

Fig. 3 After deepening of the first web space, a palmar flap (a) and a free partial skin graft (b) were used to cover the remaining gap. The hand's grip function was immediately restored postoperatively (c)

Since the first description in 1950 by Otto Hilgenfeldt of thumb reconstruction by great toe transplantation [13], many refinements and modifications of the autologous transplantation method have been evolved, in an effort to successfully address the issues related to this procedure [14].

In a case that even a small part of the first phalanx is preserved, a modified wrap-around flap $[8,14,15]$ or a twisted two toe flap as described by Fouchet $[6,14]$ would have been the treatment of choice. Both methods reduce donor site morbidity by preserving toe osseous structures, while both cosmetic and functional results were reported to be superior for toe transfer compared to pollicisation, when used for thumb reconstruction [16].

Although we agree that the modified wrap-around flap or the twisted two toe flap would have been the treatment of choice, we felt that in our case the use of an osteocutaneous vascularised flap harvested from the remaining 2nd metacarpal was justified, as the presence of the 2nd metacarpal stump posed a significant obstacle to hand function and simultaneously was an ideal donor site. Moreover, the removal of the remaining 2nd metacarpal allowed the more effective deepening of the first web space. The stable osteosynthesis performed, combined with the preserved perfusion and innervation of the neothumb, allowed the immediate postoperative restoration of its mobility and sensation. In this aspect, significant was the contribution of the retention of the proximal part of the first phalanx, as the insertion point of abductor pollicis brevis, adductor pollicis and flexor pollicis brevis muscles. The function of opponens pollicis muscle was unaffected, since this muscle inserts into the distal part of the first metacarpal.

Further advantages of this technique are the relative short surgical time and the absence of donor site morbidity, while in case of failure alternative options remain open.

As a conclusion, it seems that under proper indications, the creation of a neothumb by use of an osteocutaneous, vascularised, 2nd metacarpal flap poses a valuable alternative option with significant advantages in the treatment 

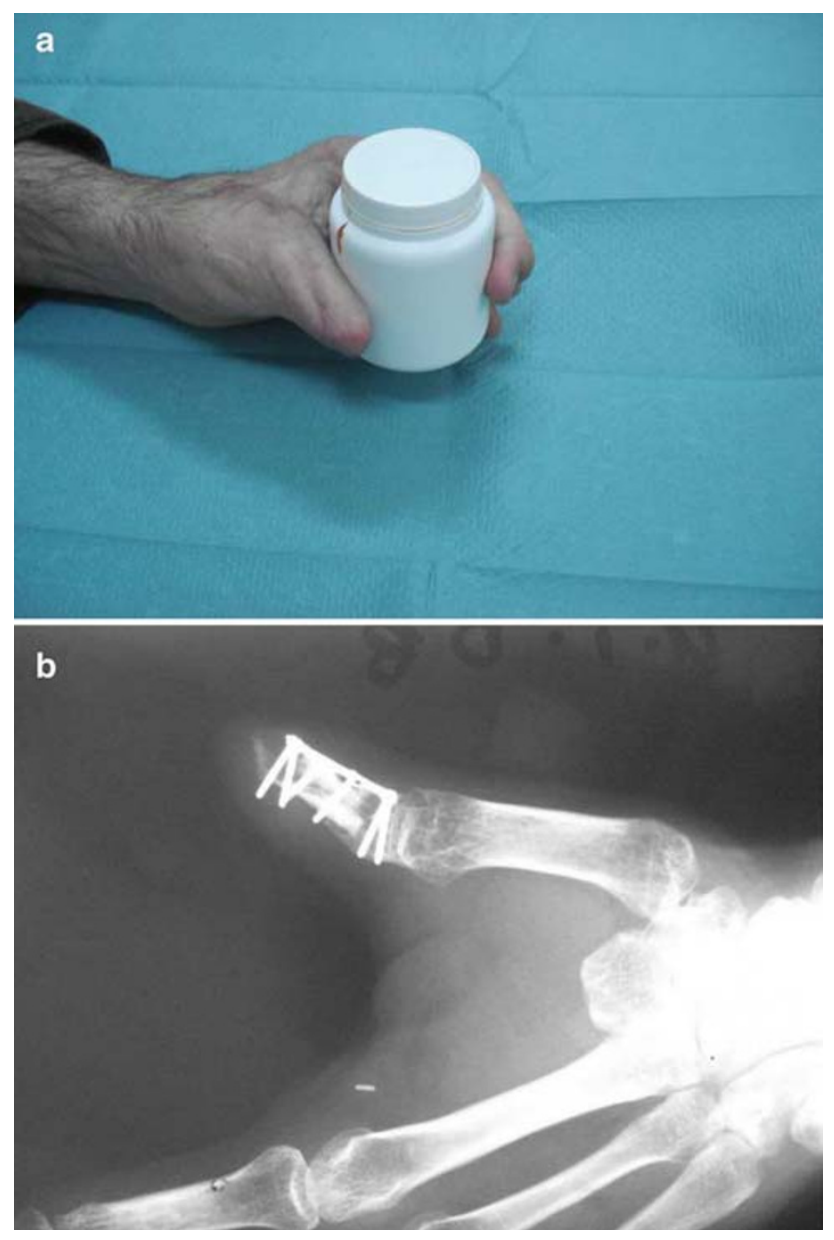

Fig. 4 Clinical and radiological appearance 14 months postoperatively

of traumatic thumb amputation, since it combines the avoidance of both complex microsurgical techniques and donor side morbidity with very good functional and aesthetic result.

\section{References}

1. Bell C (1833) The hand, its mechanism and vital endowmentsat evincing design. Pickering, London

2. Elbeshbeshy B, Paksima N (2001) Post-traumatic thumb reconstruction. Bull Hosp Jt Dis 60(3-4):130-133

3. Emerson ET, Krizek TJ, Greenwald DP (1996) Anatomy, physiology, and functional restoration of the thumb. Ann Plast Surg 36(2):180-191

4. Lister G (1985) The choice of procedure following thumb amputation. Clin Orthop Relat Res 195(May):45-51

5. Buck-Gramcko D (1981) Thumb reconstruction after amputation injuries. Handchirurgie 13(1-2):14-27

6. Foucher G, Merle M, Maneaud M, Michon J (1980) Microsurgical free partial toe transfer in hand reconstruction: a report of 12 cases. Plast Reconstr Surg 65(5):616-627

7. Foucher G, Sammut D, Citron N (1990) Free vascularized toejoint transfer in hand reconstruction: a series of 25 patients. J Reconstr Microsurg 6(3):201-207

8. Morrison WA, O'Brien BM, MacLeod AM (1980) Thumb reconstruction with a free neurovascular wrap-around flap from the big toe. J Hand Surg Am 5(6):575-583

9. Matev I (2003) Thumb metacarpal lengthening. Tech Hand Up Extrem Surg 7(4):157-163

10. Loda G (1991) The vascular rein technique: a new way for thumb reconstruction. Ann Chir Main Memb Super 10(3):251-254

11. Holevich J (1963) A new method of restoring sensibility to the thumb. J Bone Joint Surg Br 45:496-502

12. Muzaffar AR, Chao JJ, Friedrich JB (2005) Posttraumatic thumb reconstruction. Plast Reconstr Surg 116(5):103e-122e

13. Drucke D, Steinstrasser L, Langer S, Homann HH, Steinau HU, Lehnhardt M (2006) Otto Hilgenfeldt (1900 to 1983)—an unusual approach for finger reconstruction. Handchir Mikrochir Plast Chir 38(4):255-260

14. Foucher G, Binhammer P (1995) Plea to save the great toe in total thumb reconstruction. Microsurgery 16(6):373-376

15. Foucher G, Sammut D (1992) Aesthetic improvement of the nail by the "illusion" technique in partial toe transfer for thumb reconstruction. Ann Plast Surg 28(2):195-199

16. Michon J, Merle M, Bouchon Y, Foucher G (1984) Functional comparison between pollicization and toe-to-hand transfer for thumb reconstruction. J Reconstr Microsurg 1(2):103-112 